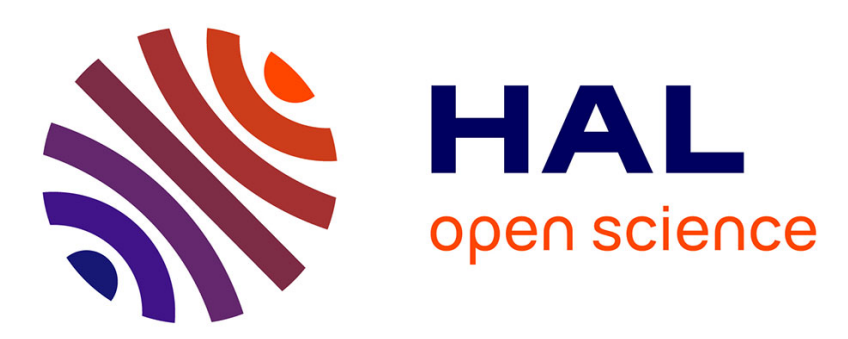

\title{
A Rapid, Deterministic Age-Depth Modeling Routine for Geological Sequences With Inherent Depth Uncertainty
}

\author{
B. Lougheed, S. Obrochta
}

\section{- To cite this version:}

B. Lougheed, S. Obrochta. A Rapid, Deterministic Age-Depth Modeling Routine for Geological Sequences With Inherent Depth Uncertainty. Paleoceanography and Paleoclimatology, 2019, 34 (1), pp.122-133. 10.1029/2018PA003457 . hal-02974751

\section{HAL Id: hal-02974751 \\ https://hal.science/hal-02974751}

Submitted on 6 May 2021

HAL is a multi-disciplinary open access archive for the deposit and dissemination of scientific research documents, whether they are published or not. The documents may come from teaching and research institutions in France or abroad, or from public or private research centers.
L'archive ouverte pluridisciplinaire HAL, est destinée au dépôt et à la diffusion de documents scientifiques de niveau recherche, publiés ou non, émanant des établissements d'enseignement et de recherche français ou étrangers, des laboratoires publics ou privés. 


\section{Paleoceanography and Paleoclimatology}

\section{RESEARCH ARTICLE \\ 10.1029/2018PA003457 \\ B. C. Lougheed and S. P. Obrochta contributed equally to this work. \\ A Rapid, Deterministic Age-Depth Modeling Routine for Geological Sequences With Inherent Depth Uncertainty}

Key Points:

- We present open source software for rapid (matter of seconds) production of age-depth models

- Interactive graphical user interface enables users to iteratively explore multiple model settings

- Age and depth uncertainty can be processed for both ${ }^{14} \mathrm{C}$ and calendar ages

Correspondence to:

B. C. Lougheed and S. P. Obrochta,

bryan.lougheed@geo.uu.se;

obrochta@gipc.akita-u.ac.jp

Citation:

Lougheed, B. C., \& Obrochta, S. P. (2019). A rapid, deterministic age-depth modeling routine for geological sequences with inherent depth uncertainty. Paleoceanography and Paleoclimatology, 34, 122-133. https:// doi.org/10.1029/2018PA003457

Received 7 AUG 2018

Accepted 18 DEC 2018

Accepted article online 2 JAN 2019

Published online 30 JAN 2019

Corrected 15 FEB 2019

This article was corrected on 15 FEB 2019. See the end of the full text for details.

\author{
B. C. Lougheed ${ }^{1,2}$ iD and S. P. Obrochta ${ }^{3}$ iD
} Akita, Japan data set to multiple parameters.

\section{Introduction}

${ }^{1}$ LSCE/IPSL, Laboratoire CNRS-CEA-UVSQ, Université Paris-Saclay, Gif-sur-Yvette, France, ${ }^{2}$ Department of Earth Sciences, Uppsala University, Uppsala, Sweden, ${ }^{3}$ Graduate School of International Resource Science, Akita University,

Abstract Traditional approaches to age-depth modeling typically assume no uncertainty for the depth value of dated intervals. However, such an assumption may not be fully valid in the case of poor coring recovery or significant sediment deformation, as well as in the case of a large subsampling interval. In consideration of these issues, we present a new age-depth modeling routine, Undatable, which includes uncertainty in both age and depth. Undatable uses Bayesian radiocarbon $\left({ }^{14} \mathrm{C}\right)$ calibration software (MatCal) and a deterministic approach with a positive sediment accumulation rate assumed a priori which, combined with efficient programming practices, allows for the rapid production (in a matter of seconds in many cases) of age-depth models for multiple types of geological archives. Undatable has so far been successfully applied to coral archives, as well as sediment archives from estuarine, lacustrine, and deep-sea environments. Through the inclusion of a bootstrapping option, the software performs particularly well in the case of a large scatter in age-depth constraints by expanding the uncertainty envelope of the age-depth model. Unlike other deterministic models, increasing the density of age-depth constraints results in increased precision in Undatable, even at centennial scale, thus emulating the results of probabilistic models. In addition to the code itself, we also provide an interactive graphical user interface (GUI) that allows users to experiment with multiple age-depth model settings to investigate the sensitivity of a given

Geological archives constitute an invaluable resource for elucidating the Earth's history. Careful reconstruction of the timing of past processes recorded by geological archives, with diligent consideration of the associated uncertainties, is crucial to understand the interdependencies and spatiotemporal characteristics of past climate processes. Geochronologies are typically derived from age estimates for discrete intervals that are then used to develop an age-depth relationship for an entire sequence (i.e., age-depth modeling). Following statistical sampling of the age-depth constraints, ages and confidence intervals are assigned to intermediate intervals lacking direct age control. These confidence intervals are often based on assumptions regarding the likelihood of possible SAR = variability between age-depth points. A basic approach assumes a more or less constant SAR uncertainty (Blaauw, 2010), while more sophisticated models may increase SAR uncertainty with distance from derived age-depth constraints, as will be discussed below.

Within the geoscience community, archeologists and palaeoecologists have been particularly active in constructing age-depth modeling routines, pioneering software for such applications. One of the first software packages developed for constructing age-depth models, the standalone OxCal (Bronk Ramsey, 1995), used statistical analysis of calibrated radiocarbon $\left({ }^{14} \mathrm{C}\right)$ age probability density functions (PDFs) to construct depositional models for sedimentary sequences, and this software has been continually updated since its first release. Subsequently developed algorithms include the open-source packages BChron (Haslett \& Parnell, 2008; Parnell et al., 2008), Clam (Blaauw, 2010), and Bacon (Blaauw \& Christen, 2011), all of which are written in the $R$ programming language.

Each type of geological archive presents unique features and challenges. Of the aforementioned software packages, Bacon and OxCal were originally developed for palaeoecological (e.g., peat sequences) and archeological sites, respectively. Consequently, these packages perform exceedingly well in these
(C)2019. American Geophysical Union. All Rights Reserved. 
environments, which are typically free from major complications due to physical and biological churning of the sediment. These models may therefore reasonably treat outlying, reversing age-depth determinations that are out of stratigraphic order very liberally, with little effect on the modeled median or the credible interval of the posterior age distribution.

However, such assumptions relating to outliers may not be fully valid in marine and lacustrine settings, particularly when the occurrence of bioturbation means that depositional layering is not preserved. Physical and biological processes may result in sediment of older age being preserved stratigraphically above younger material (due to noncomplete mixing), or even a systematic homogeneous mixed layer depth in the case of many deep-sea sediment cores (Bard et al., 1987; Berger \& Heath, 1968; Goldberg \& Koide, 1962; Lougheed et al., 2018; Peng et al., 1979; Trauth et al., 1997). While detailed sedimentological observations can often reveal the presence of heavily bioturbated or otherwise disturbed intervals, as well as the presence of turbiditic deposits, these interpretations are subjective and not always straightforward. Age-depth constraints out of stratigraphical order also appear in sequences that show no visible signs of disturbance. Furthermore, in the case of large sample sizes, averaging of the downcore signal may increase the overall uncertainty for agedepth constraints, for example, due to a systematic smoothing out of the temporal signal in the depth domain (e.g., bioturbation), which may go undetected if no age-depth reversals are present (Lougheed et al., 2018). It is therefore generally advisable to increase age and depth uncertainty to account for both the possibility of age-depth outliers and/or general increased uncertainty due to the aforementioned temporal smoothing resulting from systematic bioturbation.

In addition to the challenges posed by bioturbation, there is also an inherent depth uncertainty in the retrieval and sampling of geological archives. Technical challenges related to sediment coring may result in core recovery (the length of material recovered relative to the distance cored) that is not $100 \%$, indicating, for example, compaction during the coring processes or postrecovery expansion, with volume doubling in some extreme cases (Obrochta et al., 2017). Linear and nonlinear sediment expansion can be mathematically compensated for, at least partially (ibid.), but such a correction results in an increased depth uncertainty for age-depth constraints. Conversely, when recovery is less than $100 \%$, the recovered sequence may not be continuous and free to move within the core liner (Webster et al., 2018). Even when recovery is exactly $100 \%$, downhole logging indicates that the recovered position of stratigraphic layers is often not consistent with the in situ position. Further depth uncertainty is introduced during the subsampling process. Typically, researchers retrieve fixed volume samples integrated over fixed depth intervals, usually $1 \mathrm{~cm}$. In such a case, age-depth constraints derived from such samples will have a uniform depth uncertainty of $1 \mathrm{~cm}$. Certain analytical methods and/or environments may require even larger intervals of sediment to produce enough material for a suitable age-depth constraint, thus further increasing the associated uniform depth uncertainty.

Depth uncertainty has previously been indirectly incorporated by Heegaard et al. (2005) using a mixed-effect model that included variance in the population of datable material obtained from the same stratigraphic level and considered that vertical mixing is one contributing factor. In our previous work, we required an age-depth modeling routine with the ability to directly incorporate multiple forms of depth uncertainty. To this end, we developed the Undatable software in MATLAB, which has the ability to directly incorporate inherent depth uncertainty. This software has been successfully used in multiple study settings including marine and estuarine sediment archives (Dijkstra et al., 2018; Obrochta et al., 2017), lacustrine sediment archives (Obrochta et al., 2018), and coral archives (Webster et al., 2018). The software has continued to evolve and now allows for relatively rapid (typically several seconds or less) age-depth model development, which, combined with an interactive GUI, allows for iterative exploration of model sensitivity to multiple parameters.

The Undatable software is an open-source age-depth modeling routine based on a sediment deposition simulation built using a modular design (Figure 1) that allows for future customization and expansion. The software is developed with the aim of being able to construct age-depth models incorporating realistic errors for both age and depth. Where there is an increased disagreement among age-depth constraints, Undatable increases the uncertainty envelope of the depositional model, reflecting the increased disagreement. Undatable does include basic outlier analysis, but age-depth constraints will only be excluded as outliers in certain cases, which can be mitigated (see section 2.2). 
(a)

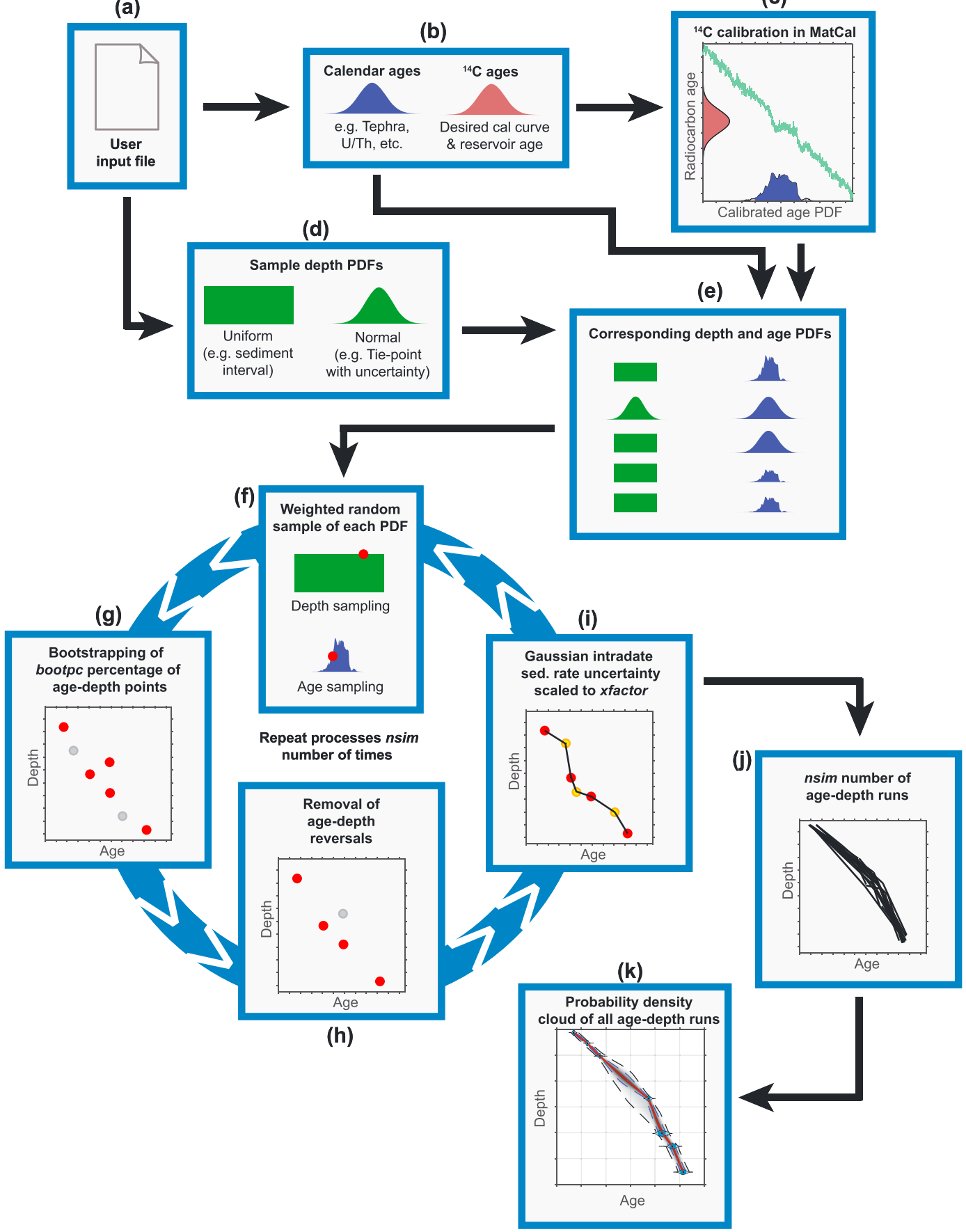

Figure 1. Flowchart overview of the processes included in Undatable. Letters a-k correspond to technical descriptions in section 2.0 of the main text.

Throughout the development and refinement of Undatable, a great emphasis has been placed upon employing efficient coding practices, leading to a software package that can produce an age-depth model with $10^{5}$ iterations in a matter of seconds using a standard personal computer. This speed will encourage users to iteratively experiment with settings to obtain experience-derived insight into the sensitivity of a particular data set to various parameters. The functioning of the Undatable age-depth modeling package is illustrated in Figure 1, and a detailed technical overview is given in section 2. The performance of the software with both synthetic and real-world data is evaluated in section 3 . 


\section{Technical Overview of the Undatable Age-Depth Modeling Routine}

\subsection{Age and Depth Uncertainty Sampling for each Age-Depth Constraint}

Age PDFs are constructed for each age-depth constraint based on the input data (Figure 1a) provided by the user. In the case of calendar ages, the mean age and associated $1 \sigma$ value provided by the user are used to construct a Gaussian distribution (Figure $1 \mathrm{~b}$ ). In the case of ${ }^{14} \mathrm{C}$ ages, the laboratory mean ${ }^{14} \mathrm{C}$ age and associated $1 \sigma$ value are calibrated to an expected calendar age distribution using the embedded Bayesian ${ }^{14} \mathrm{C}$ calibration software MatCal (Lougheed \& Obrochta, 2016; Figures $1 \mathrm{~b}$ and 1c). For these ${ }^{14} \mathrm{C}$ ages, the user may also specify a chosen calibration curve and reservoir age correction. Age PDFs are truncated to either the $2 \sigma$ confidence interval range for calendar age input or the $2 \sigma$ highest posterior density credible interval range for radiocarbon age input (due to Bayesian calibration). Truncating the annually sampled PDFs is memory efficient because otherwise each stored age PDF will cover the entire 50-ka history of the calibration curve (in the case of ${ }^{14} \mathrm{C}$ dates) or infinity (in the case of calendar ages).

We allow the user to impart depth uncertainty (Figure 1d) for an individual age-depth constraint in one of the three following ways: (1) Uncertainty may be considered as uniform by inputting a discrete sediment interval, for example, 1-2 cm; (2) Gaussian uncertainty can be assumed if a mean depth and $1 \sigma$ value are input, for example, $10 \pm 1 \mathrm{~cm}$; and (3) zero uncertainty can be specified using a sediment interval with identical upper and lower bounds, for example, $1-1 \mathrm{~cm}$. Uniform uncertainty is typical for marine and lacustrine cores where either macrofossils or bulk organic matter is subsampled from a discrete interval. Gaussian uncertainty is appropriate when aligning to a reference series (e.g., a tie-point to an event stratigraphy or isotope data set), and the exact downcore depth of the feature is unclear. No uncertainty in depth could be assumed in the case of, for example, thin terrestrial macrofossils aligned with the bedding plane.

Based on the nsim number of simulations requested by the user, each depth and age PDF are subjected to weighted random sampling nsim number of times (Figure 1f), with all sampled ages and depths being stored in memory to be used later in the sediment deposition simulation. Additionally, the user can choose to combine the age PDFs for age-depth constraints with identical depth intervals (using the combine option) into a single age PDF for that depth. This combining process is accomplished by summing the all calendar age PDFs for a particular depth, normalizing them by the sum of all elements, and carrying out highest posterior density analysis to ascertain a $2 \sigma$ credible interval. For the purposes of the age-depth modeling run, these combined age-depth constraints will then be handled as a single age-depth constraint. This combine setting is recommended and enabled by default. If the combine setting is not enabled, then only one of the age PDFs from a shared depth will be chosen (randomly) for each undatable simulation iteration.

\subsection{Sediment Deposition Simulation}

The functioning of the age-depth modeling routine is centered on a depositional model incorporating an a priori assumption that excludes the possibility of negative SAR. Based on the nsim number of simulations requested by the user (e.g., $10^{3}, 10^{4}$, and $10^{5}$ ), nsim number of unique age-depth runs are created by selecting from the previously sampled age-depth constraints (Figure 1f), sorted by their sampled depth. In each of the nsim age-depth runs, bootpc percentage of the age-depth constraints that have been selected for bootstrapping are randomly removed (Figure $1 \mathrm{~g}$ ), with the exception of the uppermost and lowermost age-depth constraint, which are not used for bootstrapping as they provide a start and end point for the age-depth simulation. Subsequently, any age-depth reversals (i.e., negative SAR) present in any of the nsim number of age-depth runs are removed in a stepwise fashion from the lowest age-depth constraint upwards, that is, in the direction of sediment deposition (Figure 1h). In cases where an age-depth constraint is out of stratigraphic order relative to the directly underlying constraint, the overlying constraint will be skipped in all $n$ sim runs if the $2 \sigma$ ranges do not overlap. This effect can be mitigated by increasing bootpc, which will increase the likelihood that the outlier's neighboring age-depth constraint within a given run does not produce an age-depth reversal.

After the completion of the aforementioned bootstrapping and reversal elimination processes, an interconnecting point is inserted between all remaining age-depth constraints in all nsim number of age-depth runs. The purpose of this interconnecting point is to take into account increased age-depth uncertainty with increasing distance from the age-depth constraints, that is, SAR uncertainty. For each required interconnecting point in each nsim number of runs, the age and depth coordinates of the interconnecting point are 
defined by weighted random sampling of Gaussian distributions drawn between the sampled ages and depths for every pair of age-depth constraints (Figure 1i; Finkenbinder et al., 2018; Marcott et al., 2013). We scale the widths of the Gaussian distributions using the xfactor input, whereby the user can increase the Gaussian SAR uncertainty between age-depth constraints by increasing the xfactor. Specifically, the Gaussian distributions are scaled by multiplying their $1 \sigma$ value by the distance between the neighboring age-depth constraints times the $x$ factor value.

\subsection{Building the Age-Depth Uncertainty Envelope}

After the production of nsim number of unique age-depth runs (Figure 1j), the next step is to calculate downcore median ages, confidence intervals, and a density cloud that displays the most likely regions of age-depth probability (Figure 1k). This process involves linearly interpolating all nsim number of unique age-depth runs to 1 depth unit resolution (e.g., $\mathrm{cm}$ ), and then computing the 1st to 99th percentiles of the ages corresponding to each depth unit. Darker regions of the density cloud are closer to the 50th percentile.

We have increased the speed of the interpolation process by a factor of 20 in comparison with the standard MATLAB interpolation scripts by outsourcing the interpolation process to an external $\mathrm{C}++$ function (nakeinterp1.c by Bruno Luong) that has been compiled to a MEX file for use within the MATLAB environment. We have included MEX files that are compatible with 64-bit versions of Windows, Mac, and Linux. Should the MEX file fail to run correctly, Undatable will detect this failure and revert to using a slower interpolation function instead.

\section{Evaluating Undatable in Practice}

\subsection{Treating Uncertainty}

Undatable offers a number of ways to treat uncertainty. The two main methods involve the settings $x$ factor and bootpc. The xfactor setting can be used to increase the SAR uncertainty between all pairs of age-depth constraints by scaling the Gaussian functions used to determine the location of the intermediate points. Essentially, a higher xfactor will lead to larger uncertainty bubbles between each pair of age-depth constraints, and vice versa. Users can input any value for $x$ factor, but values between 0.05 and 0.3 will give a broad enough range of SAR uncertainty suitable for most users. The age-depth models published to date using Undatable have employed in $x$ factor value of 0.1 . The other main way to treat uncertainty is through bootpc, the percentage of age-depth constraints to be bootstrapped (i.e., randomly removed) in each agedepth model iteration. A higher bootpc will cause the final age-depth model to explore a larger number of routing possibilities (due to passing through a different combination of age-depth constraints in every iteration), which will result in a wider uncertainty envelope over the entire age-depth run. The bootpc setting is especially useful when dealing with a large scatter in age-depth constraints. A visual demonstration of the interaction between bootpc and $x$ factor is shown in Figure 2 using synthetic age-depth constraints. In this demonstration, it can be seen how xfactor alone serves to increase the SAR uncertainty between age-depth constraints (Figures 2a, 2b, and 2c). By itself, bootpc can help to increase uncertainty to take into account increase scatter in age-depth constraints (Figures 2a, 2d, and 2e). An age-depth model optimized to the particular data set under consideration can be obtained by iteratively adjusting these two parameters (Figures 2a-2i).

Additionally, it is also possible to selectively exclude certain age-depth constraints from the bootstrapping process. This possibility is especially useful in the case of stratigraphically and chronologically well-defined age-depth constraints, such as tephra layers, and can be specified in the input file (or in the GUI) by the user for each age-depth constraint. For example, considering a data set with 10 age-depth constraints, one of which is a tephra layer, a user could choose to apply $33 \%$ bootstrapping ( bootpc $=33$ ), but with the tephra layer excluded from the bootstrapping process. In such a case, all age-depth iterations would contain the tephra layer plus six additional age-depth constrains randomly selected from the remaining nine $(9-[9 \times 0.33])$, for a total of seven age-depth constraints per iteration (i.e., rounded to the nearest whole number). Each age-depth iteration will be routed through the tephra layer, thus weighting the final agedepth model toward the tephra layer. 


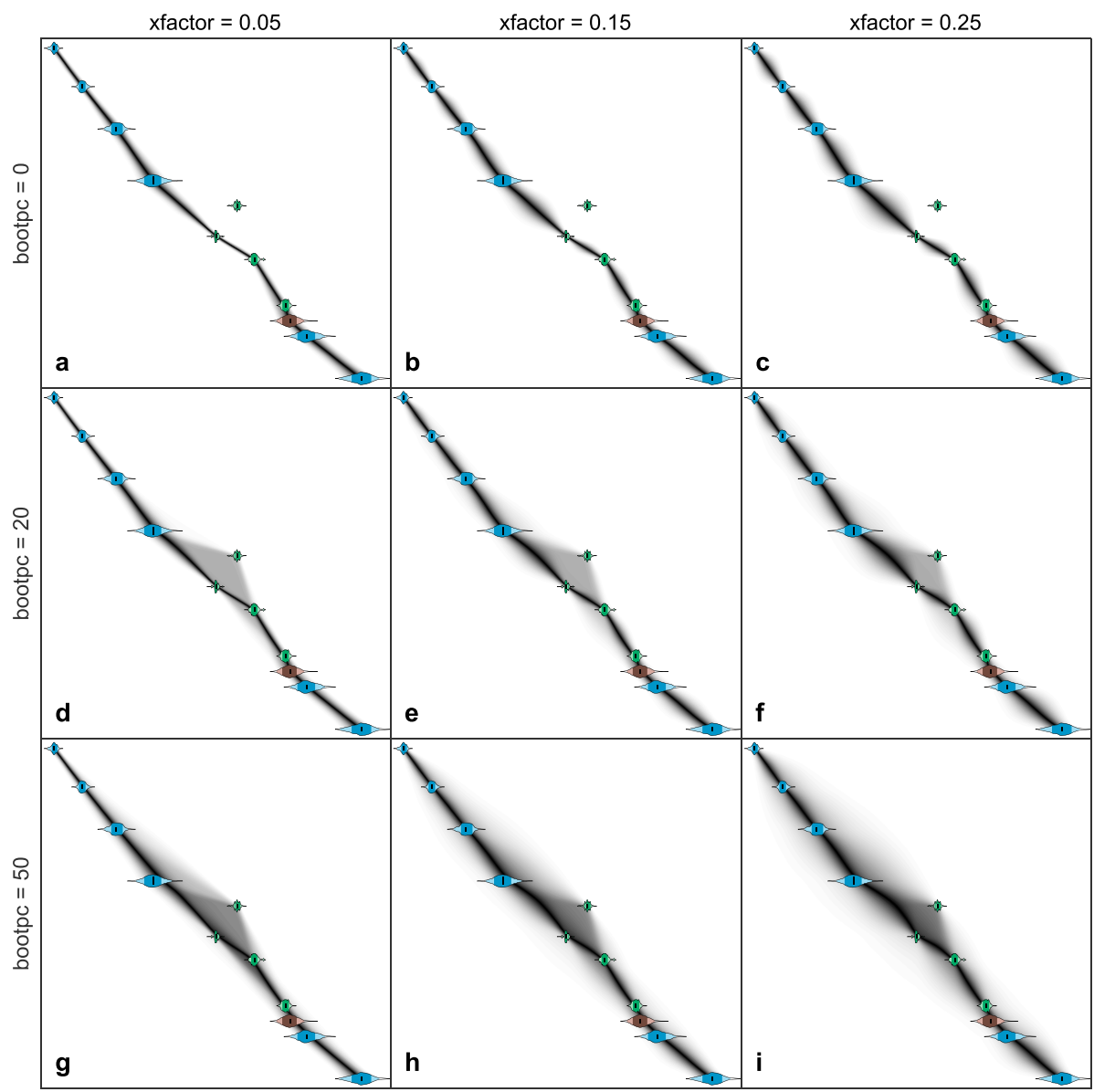

Figure 2. Overview of nine different age-depth model scenarios using the exact same synthetic age-depth constraints as input, but with different bootpc and xfactor settings. All age-depth models have been run using $10^{5}$ Monte Carlo iterations. Colored probability density functions illustrate the synthetic age-depth constraints. The grey cloud indicates the probability density cloud of the age-depth model, whereby darker colors indicate higher age-depth probability. Colors of probability density functions represent different types geochrological input (green $={ }^{14} \mathrm{C}$ terrestrial, blue $={ }^{14} \mathrm{C}$ marine, brown $={ }^{14} \mathrm{C}$ bulk sediment). The settings used for the different age-depth model scenarios are (a) bootpc $=0$, xfactor $=0.05$; (b) bootpc $=0$, xfactor $=0.15$; (c) bootpc $=0$, xfactor $=0.25$; (d) bootpc $=20$, xfactor $=0.05$; (e) bootpc $=20$, xfactor $=0.15 ;(\mathrm{f})$ bootpc $=20$, xfactor $=0.25 ;(\mathrm{g})$ bootpc $=50$, xfactor $=0.05 ;(\mathrm{h})$ bootpc $=50$, xfactor $=0.15$; and $(\mathrm{i})$ bootpc $=50$, xfactor $=0.25$.

\subsection{Running Undatable on Real World Archives}

\subsubsection{Cariaco Basin}

Using the Cariaco Basin (Ocean Drilling Program [ODP] Site 1002) foraminifera record (466 ${ }^{14} \mathrm{C}$ age-depth constraints; Hughen et al., 2006), we examine the ability of Undatable to deal with a very large data set containing intervals of varying scatter in age-depth constraints. In this case, when bootpc is set to a lower percentage (10\%), the positive SAR assumed a priori in Undatable causes the age-depth model to constantly route through isolated, significantly younger age-depth constraints, which appear to be outliers relative to the adjacent constraints, resulting in a step-like age-depth model (Figure 3a). When bootpc is sufficiently increased (to 80\%), these isolated age-depth constraints are excluded from a sufficient number of model iterations such that their contribution to the median route of the age-depth model is reduced (Figure $3 b$ ). In compensation, the overall uncertainty on the age-depth model increases, so that the existence of the outliers is still acknowledged by the uncertainty envelope. Users should take caution in selecting an appropriate bootpc; in the case of the Cariaco Basin record, both the absolute number $(n=466 ; n=320$ after combining constraints from the same depth interval) and temporal density of ${ }^{14} \mathrm{C}$ age-depth constraints are of centennial scale; thus, it possible to use a relatively high bootpc. Were one to apply a bootpc of $80 \%$ to a record with, 

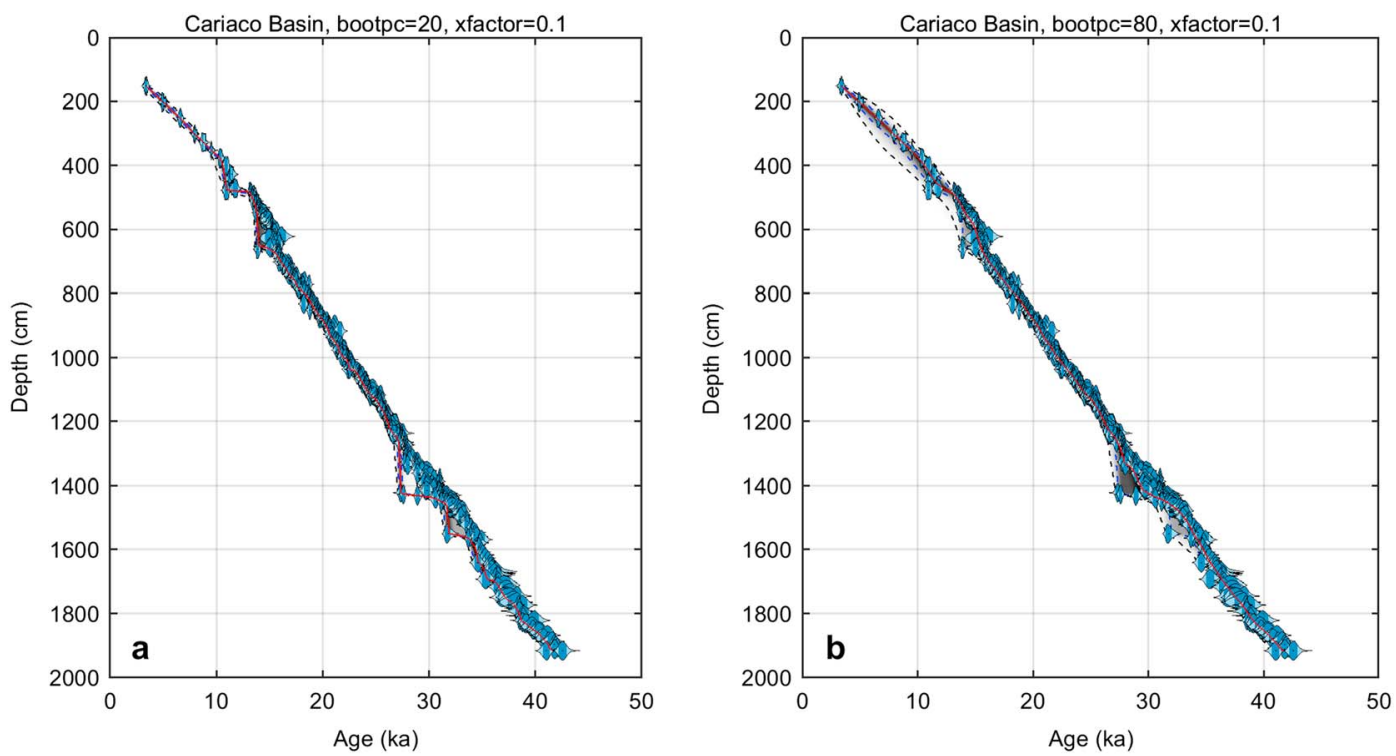

Figure 3. Comparison of two age-depth model scenarios applied to the same Cariaco Basin (Ocean Drilling Program [ODP] Site 1002) high-density radiocarbon age-depth constraints (Hughen et al., 2006). On the left (a) a bootpc of 20 and xfactor of 0.1 are applied. On the right (b) a bootpc of 80 and xfactor of 0.1 are applied. Blue probability density functions indicate the radiocarbon age-depth constraints. The grey cloud indicates the probability density cloud of the agedepth model, whereby darker colors indicate higher age-depth probability. The red line indicates the age-depth model median. The dashed black and blue lines indicate the age-depth model $2 \sigma$ and $1 \sigma$ confidence intervals, respectively. For more detailed discussion, see section 3.1 in text.

for example, only 10 age-depth constraints in total, then each model iteration would contain only two agedepth constraints, likely resulting in an unrealistic age-depth model. As a general rule, one can consider attempting a higher bootpc for records with higher absolute numbers of age-depth constraints, and vice versa. In any case, users are encouraged to take advantage of the speed of Undatable to iteratively experiment with multiple bootpc settings.

\subsubsection{Lake Motosu}

As previously mentioned, Undatable offers the ability to weight the final age-depth model toward age-depth constraints of higher quality, that is, tephra layers and/or terrestrial ${ }^{14} \mathrm{C}$ dates. We demonstrate this ability using a lacustrine sediment sequence recovered Lake Motosu (one of the Fuji Five Lakes) in Japan. Site MOT15-2 is an 3.5-m long, nonlaminated sequence with 31 age-depth constraints developed using multiple methods with varying accuracy and precision, namely, two widespread marker tephras of independently constrained age, five terrestrial macrofossil ${ }^{14} \mathrm{C}$ ages, and 24 bulk organic matter ${ }^{14} \mathrm{C}$ ages (Obrochta et al., 2018). While the dated organic matter is of primarily lacustrine origin (as evidenced by relatively low $\mathrm{C} / \mathrm{N}$ ratios), the lake appears to have a time-variable reservoir age, subjecting the bulk dates to increased uncertainty. In this example (Figure 4a), we take advantage of the selective bootstrapping function of Undatable to anchor the age-depth model using the more accurate terrestrial macrofossil ${ }^{14} \mathrm{C}$ and tephra age-depth constraints by selectively excluding these from the bootstrapping process, while the bulk sediment ${ }^{14} \mathrm{C}$ agedepth constraints are included in the bootstrapping process, with bootpc set to $65 \%$.

The interval at $\sim 2.75 \mathrm{~m}$ contains several age-depth constraints that are not in stratigraphic order, but there is no evidence of reworking or other justifiable means for excluding them from the model (e.g., AMS condition). The selective bootstrapping process increases the uncertainty envelope to take into account the presence of increased age-depth constraint scatter at the aforementioned interval. The uncertainty envelope for an underlying interval is also increased due to a combination of lower-density dating and a SAR inflection point (Figure 4a). For other intervals, where terrestrial macrofossil ${ }^{14} \mathrm{C}$ and tephra ages are available, the uncertainty envelope is smaller. We consider that this selective behavior realistically reflects the varying agedepth uncertainty at different intervals of the sediment sequence. By contrast, Bacon produces a much narrower error envelope, narrower in fact than individual organic matter calendar age PDFs, particularly at 

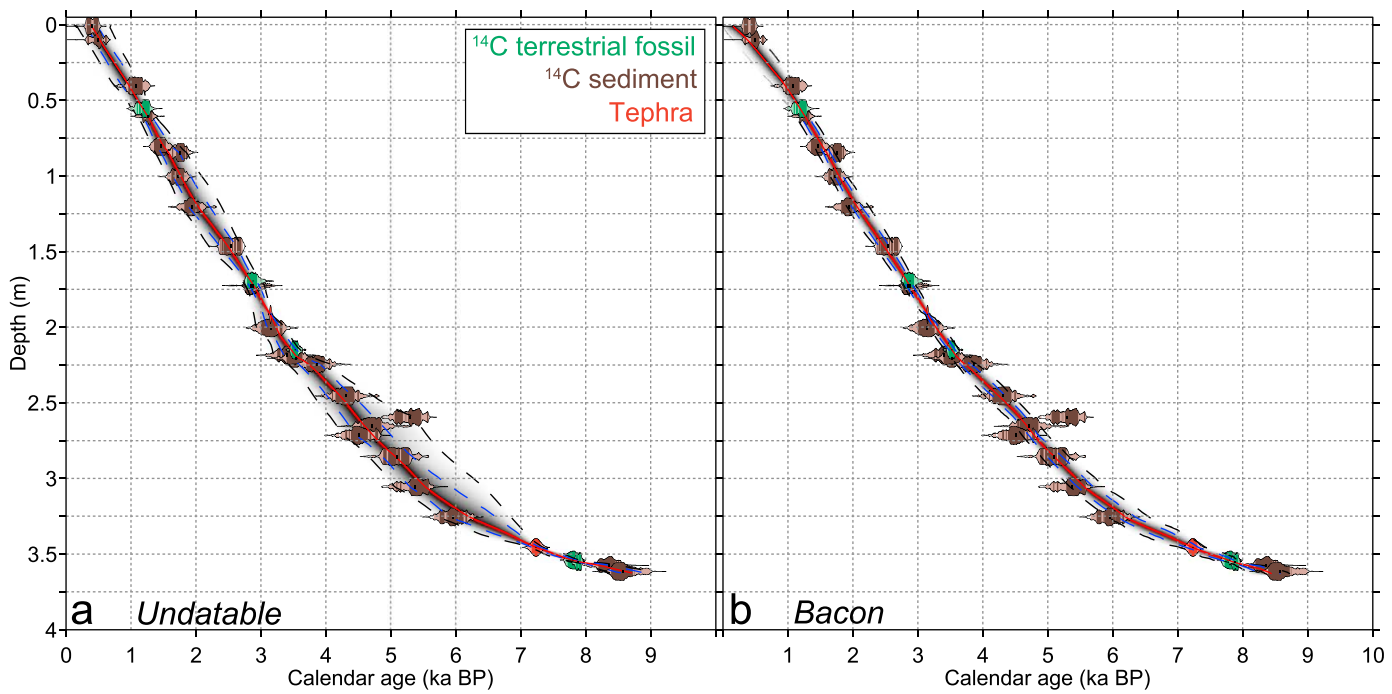

Figure 4. (a) Undatable age-depth model for Site MOT15-2 from Lake Motosu, Japan. The red line is median, and the blue and black broken lines represent $1 \sigma$ and $2 \sigma$ confidence intervals, respectively. (b) Bacon age-depth model using the same age determinations and run from 0 to $363 \mathrm{~cm}$ at $1-\mathrm{cm}$ resolution with a mean accumulation rate of 40 , accumulation shape of 1.05 , memory set to 0.9 , and a memory strength of 20 .

both the SAR inflection point and the interval containing the outlier (Figure 4b; see figure caption for Bacon settings). Except for the upper and lowermost intervals, where the Bacon-derived median drifts away from the age-depth constraints (Figure 4b), we note that the median age-depth relationship obtained by both models is not significantly different (Figure 5a). Away from the edges of the data set, maximum offset between Undatable and Bacon is $\sim 80$ years (Figure $5 \mathrm{~b}$ ). The offset is entirely contained within the Undatable $1 \sigma$ range and the Bacon $2 \sigma$ range. The offset is also largely within the Bacon $1 \sigma$, only exceeding it at $\sim 2 \mathrm{~m}$. As a consequence, the selection of model software appears to not affect interpretations based solely on the median modeled age, but consideration of the full uncertainty envelope may lead to a different interpretation.
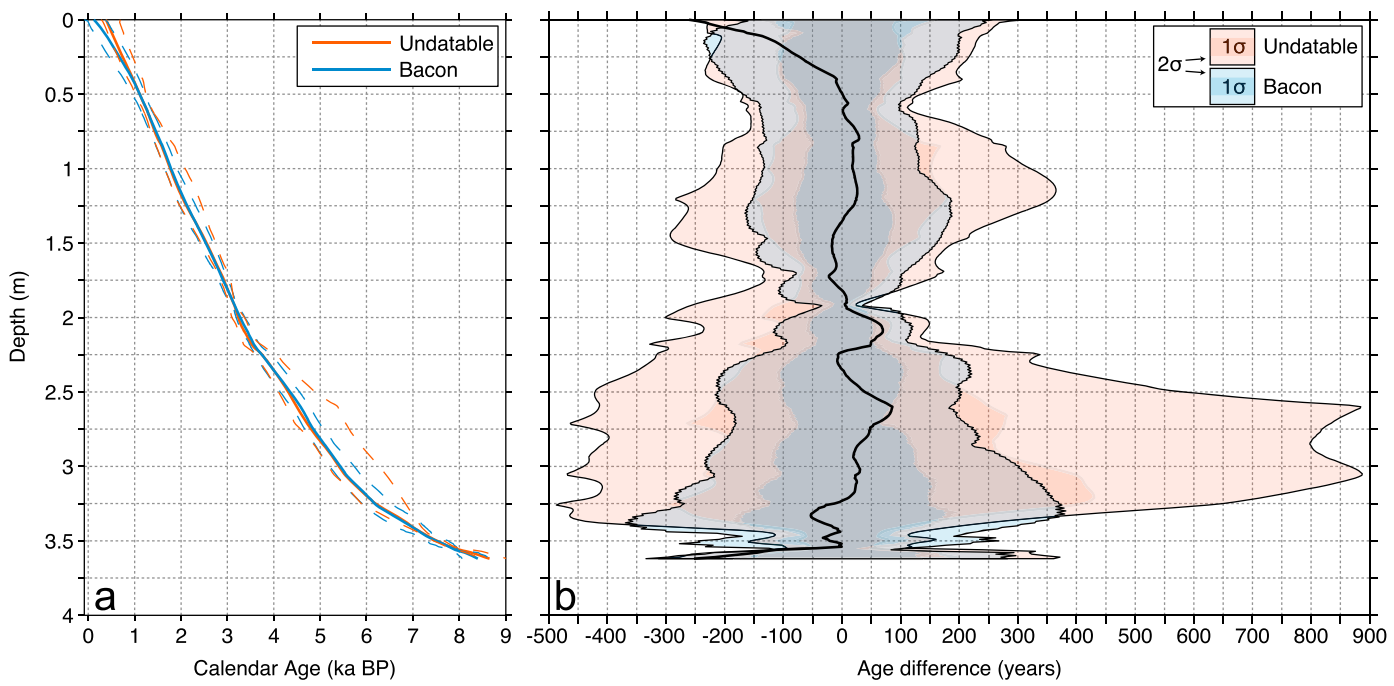

Figure 5. (a) Median, $1 \sigma$, and $2 \sigma$ for Undatable (red) Bacon (blue). (b) The Undatable median age is subtracted from the Bacon median and shown with the $1 \sigma$ and $2 \sigma$ envelopes for Undatable and Bacon. The maximum offset of $\sim 80$ years occurs at $\sim 2.25 \mathrm{~m}$ and is within both the Undatable and Bacon $1 \sigma$ ranges. Except for the upper and lower most intervals and a $\sim 20-\mathrm{cm}$ interval just below $2 \mathrm{~m}$, the offset is entirely within the Bacon $2 \sigma$ range. 

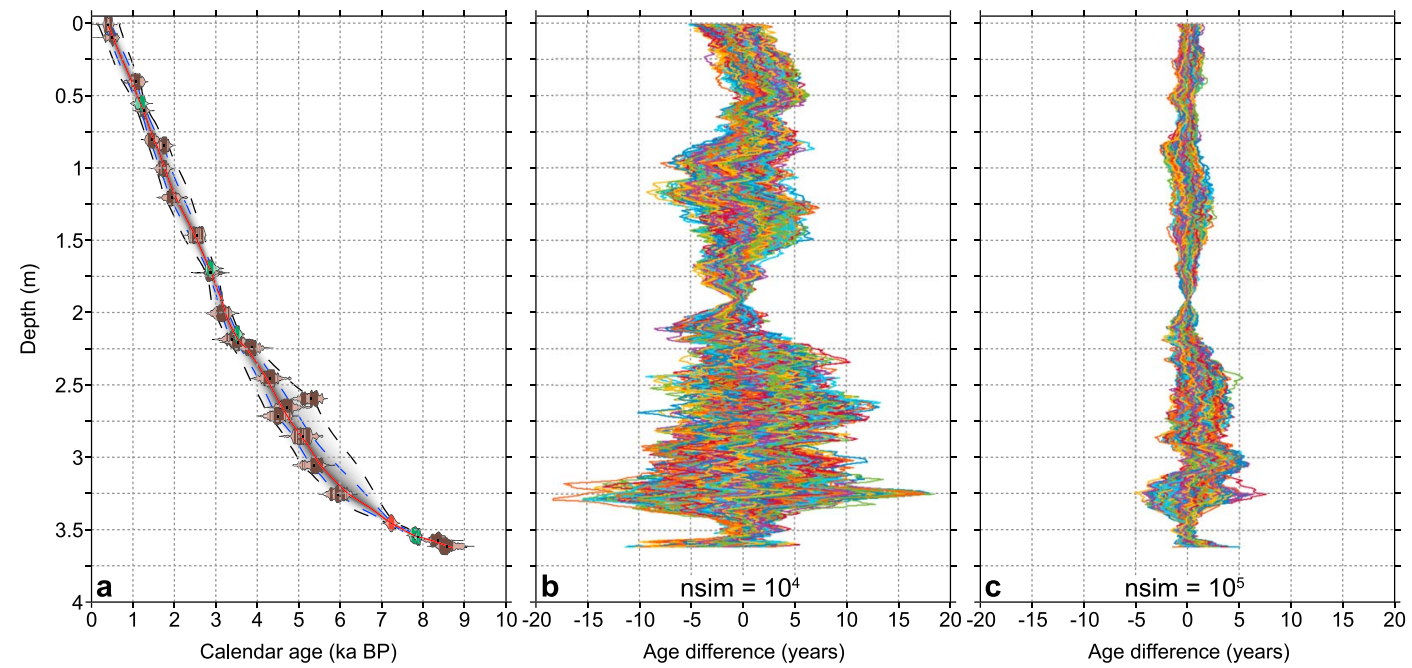

Figure 6. (a) Undatable age-depth model for Site MOT15-2 from Lake Motosu, Japan. (b) After an initial model was produced using $10^{4}$ iterations, 999 additional age models were created and subtracted from the initial model. Maximum difference is $\sim \pm 15$ years. (c) Same as (b) but using $10^{5}$ iterations. Maximum difference decreases by a factor of $\sim 3$ to $\sim \pm 5$ years.

\subsection{Reproducibility of Age-Depth Model Runs}

The Lake Motosu record is also used to explore model sensitivity to the number of iterations (nsim) and as to how this number affects Undatable's age-depth model reproducibility. One thousand age-depth models were created using nsim set to both $10^{4}$ and $10^{5}$, and the difference between the initial result and each subsequent result calculated at $1-\mathrm{cm}$ intervals. We find that the maximum difference between subsequent runs decreases by a factor of 3 as the number of age-depth model iterations is increased by one order of magnitude (Figure 6), and this difference is typically $<10$ years. As this improved reproducibility was obtained by only adding a couple of seconds to the calculation time, we recommend using $10^{5}$ simulations. A higher number of simulations $\left(10^{6}\right)$ is also possible, but when using a higher number of simulations (i.e., $10^{6}$ ) combined with many age-depth inputs (such as, e.g., Cariaco Basin), the user should be aware that Undatable may require virtual memory on some consumer-level computers, which will markedly increase calculation time.

The largest differences are observed at the first and last age-depth constraint, as well as at the major SAR inflection point at a depth of $3.75 \mathrm{~m}$. This is also the interval of maximum standard deviation, which is 1.7 years $\left(\mathrm{nsim}=10^{5}\right)$. Intervals containing bulk organic matter dates, as well as intervals where the bootstrapped bulk dates are in close proximity to macrofossils also exhibit decreased reproducibility, although the differences are still generally a decade or less. Reproducibility, in general, decreases in intervals with relatively increased model uncertainty.

\subsection{Increased Dating Density Leads to Improved Precision}

Recent work by Blaauw et al. (2018) demonstrates that increasing the number of age-depth constraints input into the BChron and Bacon age-depth modeling software leads to improvement in the overall precision on the derived age-depth model. We investigate if Undatable also exhibits similar performance using the aforementioned Cariaco Basin data set as an example. Using a bootpc of $60 \%$ and $n \operatorname{sim}$ of $10^{5}$, we carried out repeat runs while incrementally increasing dating density, starting with only six age-depth constraints. The initial age-depth constraints are at the top and bottom of the data set with an additional, four equidistantly spaced constraints. After each age-depth model was completed, mean $1 \sigma$ precision was calculated, an additional date was randomly added, and the process repeated until all 320 age-depth constraints were used in the model. This experiment was repeated a total of 500 times in eight parallel instances of MATLAB launched with GNU Parallel (Tange, 2011), for a total of 157,500 age-depth models. Results are shown as the mean 


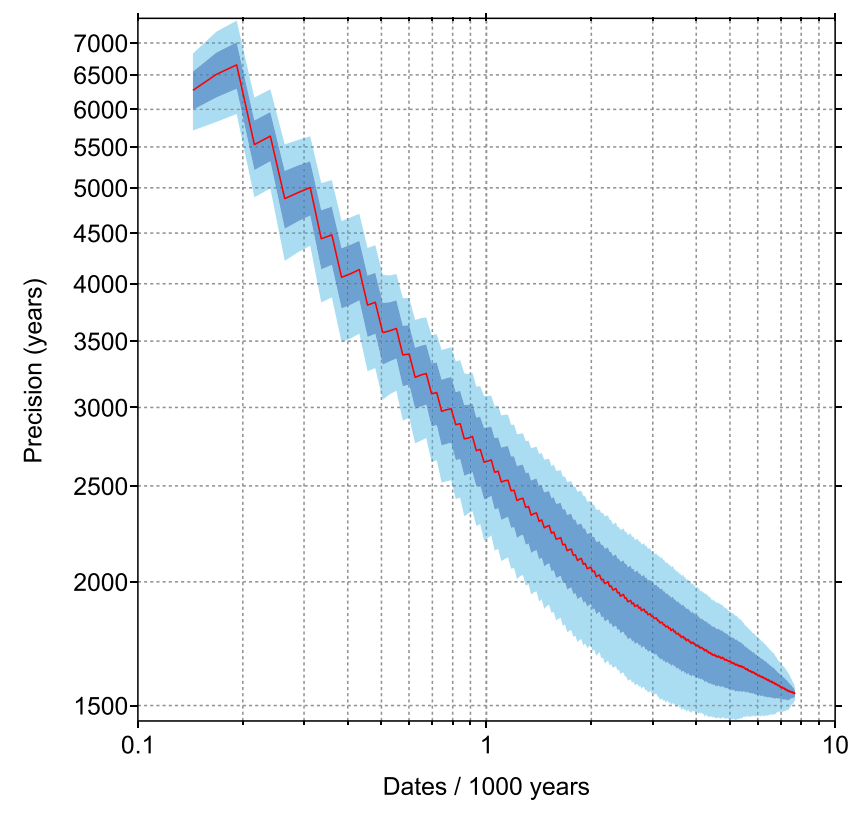

Figure 7. Result of sequentially increasing dating density at Ocean Drilling Program (ODP) Site 1002 in the Cariaco Basin. Result is the mean of 500 sets of runs starting with only six age-depth determinations and finishing with 320 (the full data set with duplicates combined). The 68.2 and 95.4 percentiles are shown in dark and light shading, respectively. Model precision continually improves with increasing dating density. and standard deviation of the 500 runs (Figure 7). Dating density ranges from 0.14 to 7.7 dates per millennium (dpm), and the precision exhibits a fivefold overall improvement at maximum dpm. Notably, Undatable does not saturate as dpm is increased in the Cariaco data set. This behavior of the Undatable age-depth modeling software is similar to that reported for BChron and Bacon (Blaauw et al., 2018).

\section{Recommended Parameter Settings}

We recommend using as large an $n$ sim as possible and find that $10^{5}$ iterations provide good repeatability with no substantial increase in calculation time relative to $10^{4}$. We also recommend using the combine feature when multiple constraints exist for the same depth level. Furthermore, as discussed in section 2.1, the user should select a depth uncertainty that is appropriate to their particular age-depth constraints.

The xfactor and bootpc parameters are more subjective and depend heavily on the nature of the data set, and the user must therefore evaluate whether values are appropriate. We find that xfactor values in the range of 0.05 to 0.3 obtain results that (subjectively) appear realistic, and the age-depth models published thus far using Undatable have employed an xfactor of 0.1. If there is independent evidence of pronounced uncertainty in SAR, then a higher value would be appropriate. Given the interplay between bootpc and xfactor (Figure 2), lower values may be appropriate when a large bootpc value is used because SAR uncertainty, by design, is proportional to the distance between two age-depth constraints. This distance will increase when bootstrapping removes more age-depth points from each iterations.

Selection of bootpc is highly dependent on the absolute number of dates, density of dating, presence/absence of age reversals, and the consistency with which the dates are distributed in the archive. In the examples provided here, we used a bootpc value of 50 in the case of 31 dates over $\sim 3.5 \mathrm{~m}$ and a value of 80 for 320 dates over $\sim 20 \mathrm{~m}$. The user should consider the number of dates that will be included in each run and ensure that a sufficient number remain to produce a reasonable age model during each simulation iteration. As the absolute number of dates in a data set increase, it is appropriate to increase bootpc, particularly if age reversals are present. However, for a data set with a high density of nonreversing dates of narrow probability range, a relatively small value could be appropriate since the data set is well constrained. If the age-depth constraints are not well distributed throughout the archive, then a high bootpc could result in a large distances with no agedepth information. In this case, the user may choose to selectively excluding key anchor dates from the bootstrapping process, as described in section 3.2.1.

\section{Outlook}

The modular and open-source construction of Undatable allows for the future addition of further features suitable to specific geological environments. For example, in the case of deep-sea sediment archives, it is possible to apply a bioturbation term a priori to age PDFs that is consistent with long-established understanding of bioturbation (Berger \& Heath, 1968; Goldberg \& Koide, 1962; Peng et al., 1979). This type of bioturbation understanding has already been successfully included in simulations of deep-sea sediment archives (Dolman \& Laepple, 2018; Löwemark et al., 2008; Trauth, 2013). Simulated effects of bioturbation have also recently shown to be in agreement with single foraminifera ${ }^{14} \mathrm{C}$ data (Lougheed et al., 2018). The inclusion of bioturbation a priori would produce broader age PDFs for lower SAR sediment cores, thus taking into account the fact that discrete depth intervals from lower SAR sediment archives must contain a systematically larger range of sediment age than discrete depth intervals from higher SAR sediment archives. This fact is currently not considered in age-depth modeling; that is, in the absence of any age-depth reversals, both a low and high SAR sediment core would produce the same geochronological uncertainty using longstanding methods. We propose that a function can be developed that uses a priori estimates of SAR and sediment mixed layer depth 
to estimate the true age distribution of a particular age-depth constraint. Such an approach would be especially useful for low SAR archives, such as deep-sea sediment cores.

It may also be possible to include a module that allows for the incorporation of SAR correction using Th normalization, in particular by integrating existing MATLAB routines designed for such a purpose (Bourne et al., 2012). The open-source nature of Undatable also allows users to implement further, minor, tweaks to certain modules of the code to suit their own needs.

\section{Conclusions}

Undatable provides a rapid and deterministic method with which to develop an age-depth model for a variety of geological archives. The speed of Undatable, combined with a GUI, also allows for users to experiment with multiple age-depth model settings on-the-fly, which enables users to develop experience-based insight into the sensitivity of their particular data set to the age-depth model parameters. The software is particularly useful for archives with high uncertainty, especially in the case of depth uncertainty or large scatter of agedepth constraints. We would encourage users to use Undatable to complement existing age-depth modeling software such as Bchron, Bacon, and OxCal and to consider which age-depth modeling software best suits the particular geological data set that they are investigating. Furthermore, we encourage the community to take advantage of the open source code we have provided to help to improve and/or customize the software in the future.

Acknowledgments

The latest version of the Undatable software and user manual can be downloaded from the Zenodo public repository at https://doi.org/10.5281/ zenodo.2527641. This work is a contribution to ERC project ACCLIMATE; the research leading to these results has received funding from the European Research Council under the European Union's Seventh Framework Programme (FP7/20072013)/ERC grant agreement 339108. B. C. L. acknowledges Swedish Research Council (Vetenskapsrådet) grant 6372014-499. Development of Undatable and radiocarbon dating at Lake Motosu were partially funded by JSPS KAKENHI 16K05571. Lake Motosu cores were obtained as part of the QuakeRecNankai project, funded by the Belgian Science Policy Office. We thank Yusuke Yokoyama and Yosuke Miyairi of The University of Tokyo Atmosphere and Ocean Research Institute for performing a great many radiocarbon dates that further contributed to model development. Philipp Kempf and Jonathan Tyler are thanked for assistance with the Bacon age model Jody Webster, Brett Metcalfe, Claire Waelbroeck, Marc De Batist, Aurélia Hubert-Ferrari, and Laura Lamair are thanked for assistance in user testing. We thank Szilárd Fazekas for advice on vectorization. Jonathan Tyler, Ian Bailey, and Claire Waelbroeck provided helpful comments on preliminary versions of the manuscript. Editor Ellen Thomas, an associate editor, and two anonymous reviewers are thanked for their very helpful reviews of our work, which helped to improve the final product.

\section{References}

Bard, E., Arnold, M., Duprat, J., Moyes, J., \& Duplessy, J. C. (1987). Reconstruction of the last deglaciation: Deconvolved records of $\delta 18$ O profiles, micropaleontological variations and accelerator mass spectrometric $14 \mathrm{C}$ dating. Climate Dynamics, 1(2), 101-112. https://doi. org/10.1007/BF01054479

Berger, W. H., \& Heath, G. R. (1968). Vertical mixing in pelagic sediments. Journal of Marine Research, 26, 134-143.

Blaauw, M. (2010). Methods and code for 'classical' age-modelling of radiocarbon sequences. Quaternary Geochronology, 5(5), 512-518. https://doi.org/10.1016/j.quageo.2010.01.002

Blaauw, M., \& Christen, J. A. (2011). Flexible paleoclimate age-depth models using an autoregressive gamma process. Bayesian Analysis, 6(3), 457-474. https://doi.org/10.1214/11-BA618

Blaauw, M., Christen, J. A., Bennett, K. D., \& Reimer, P. J. (2018). Double the dates and go for Bayes-Impacts of model choice, dating density and quality on chronologies. Quaternary Science Reviews, 188, 58-66. https://doi.org/10.1016/j.quascirev.2018.03.032

Bourne, M. D., Thomas, A. L., Mac Niocaill, C., \& Henderson, G. M. (2012). Improved determination of marine sedimentation rates using ${ }^{230} \mathrm{Th}_{x s}$. Geochemistry, Geophysics, Geosystems, 13, Q09017. https://doi.org/10.1029/2012GC004295

Bronk Ramsey, C. (1995). Radiocarbon calibration and analysis of stratigraphy; the OxCal program. Radiocarbon, 37(2), 425-430. https:// doi.org/10.1017/S0033822200030903

Dijkstra, N., Quintana Krupinski, N. B., Yamane, M., Obrochta, S. P., Miyairi, Y., Yokoyama, Y., \& Slomp, C. P. (2018). Holocene refreshening and reoxygenation of a Bothnian Sea Estuary led to enhanced phosphorus burial. Estuaries and Coasts, 41(1), $139-157$. https://doi.org/10.1007/s12237-017-0262-x

Dolman, A. M., \& Laepple, T. (2018). Sedproxy: A forward model for sediment archived climate proxies. Climate of the Past Discussions, 1-31. https://doi.org/10.5194/cp-2018-13

Finkenbinder, M. S., Abbott, M. B., Stoner, J. S., Ortiz, J. D., Finney, B. P., Dorfman, J. M., \& Stansell, N. D. (2018). Millennial-scale variability in Holocene aquatic productivity from Burial Lake, Arctic Alaska. Quaternary Science Reviews, 187, 220-234. https://doi.org/ 10.1016/j.quascirev.2018.03.019

Goldberg, E. D., \& Koide, M. (1962). Geochronological studies of deep sea sediments by the ionium/thorium method. Geochimica et Cosmochimica Acta, 26(3), 417-450.

Haslett, J., \& Parnell, A. (2008). A simple monotone process with application to radiocarbon-dated depth chronologies. Journal of the Royal Statistical Society: Series C: Applied Statistics, 57(4), 399-418. https://doi.org/10.1111/j.1467-9876.2008.00623.x

Heegaard, E., Birks, H. J. B., \& Telford, R. J. (2005). Relationships between calibrated ages and depth in stratigraphical sequences: An estimation procedure by mixed-effect regression. The Holocene, 15(4), 612-618. https://doi.org/10.1191/0959683605hl836rr

Hughen, K., Southon, J., Lehman, S., Bertrand, C., \& Turnbull, J. (2006). Marine-derived 14C calibration and activity record for the past 50,000 years updated from the Cariaco Basin. Quaternary Science Reviews, 25(23-24), 3216-3227. https://doi.org/10.1016/j.quascirev.2006.03.014

Lougheed, B. C., Metcalfe, B., Ninnemann, U. S., \& Wacker, L. (2018). Moving beyond the age-depth model paradigm in deep sea palaeoclimate archives: Dual radiocarbon and stable isotope analysis on single foraminifera. Climate of the Past, 14, 1-16. https://doi. org/10.5194/cp-2017-119

Lougheed, B. C., \& Obrochta, S. P. (2016). MatCal: Open source Bayesian ${ }^{14} \mathrm{C}$ age calibration in MATLAB. Journal of Open Research Software, 4. https://doi.org/10.5334/jors.130

Löwemark, L., Konstantinou, K. I., \& Steinke, S. (2008). Bias in foraminiferal multispecies reconstructions of paleohydrographic conditions caused by foraminiferal abundance variations and bioturbational mixing: A model approach. Marine Geology, 256(1-4), 101-106. https://doi.org/10.1016/j.margeo.2008.10.005

Marcott, S. A., Shakun, J. D., Clark, P. U., \& Mix, A. C. (2013). A reconstruction of regional and global temperature for the past 11,300 years. Science, 339(6124), 1198-1201. https://doi.org/10.1126/science.1228026

Obrochta, S. P., Andrén, T., Fazekas, S. Z., Lougheed, B. C., Snowball, I., Yokoyama, Y., et al. (2017). The undatables: Quantifying uncertainty in a highly expanded Late Glacial-Holocene sediment sequence recovered from the deepest Baltic Sea basin-IODP Site M0063. Geochemistry, Geophysics, Geosystems, 18, 858-871. https://doi.org/10.1002/2016GC006697 
Obrochta, S. P., Yokoyama, Y., Yoshimoto, M., Yamamoto, S., Miyairi, Y., Nagano, G., et al. (2018). Mt. Fuji Holocene eruption history reconstructed from proximal lake sediments and high-density radiocarbon dating. Quaternary Science Reviews, 200, 395-405. https://doi org/10.1016/j.quascirev.2018.09.001

Parnell, A. C., Haslett, J., Allen, J. R. M., Buck, C. E., \& Huntley, B. (2008). A flexible approach to assessing synchroneity of past events using Bayesian reconstructions of sedimentation history. Quaternary Science Reviews, 27(19-20), 1872-1885. https://doi.org/10.1016/j. quascirev.2008.07.009

Peng, T.-H., Broecker, W. S., \& Berger, W. H. (1979). Rates of benthic mixing in deep-sea sediment as determined by radioactive tracers Quaternary Research, 11(1), 141-149. https://doi.org/10.1016/0033-5894(79)90074-7

Tange, O. (2011). GNU parallel-The command-line power tool. The USENIX Magazine, 36(1), 42-47.

Trauth, M. H. (2013). TURBO2: A MATLAB simulation to study the effects of bioturbation on paleoceanographic time series. Computers \& Geosciences, 61, 1-10. https://doi.org/10.1016/j.cageo.2013.05.003

Trauth, M. H., Sarnthein, M., \& Arnold, M. (1997). Bioturbational mixing depth and carbon flux at the seafloor. Paleoceanography, 12(3), 517-526. https://doi.org/10.1029/97PA00722

Webster, J. M., Braga, J. C., Humblet, M., Potts, D. C., Iryu, Y., Yokoyama, Y., et al. (2018). Response of the Great Barrier Reef to sea-level and environmental changes over the past 30,000 years. Nature Geoscience, 11(6), 426-432. https://doi.org/10.1038/s41561-018-0127-3

\section{Erratum}

In the originally published version of this article, Figure 4 was published incorrectly. This error has since been corrected, and this version may be considered the authoritative version of record. 